\title{
Photo-Induced Antitumor Effect of 3,6-Bis(1-methyl-4-vinylpyridinium) Carbazole Diiodide
}

\author{
Ya-Shuan Chou, ${ }^{1}$ Cheng-Chung Chang, ${ }^{2}$ Ta-Chau Chang, ${ }^{3}$ Tsung-Lin Yang, \\ Tai-Horng Young, ${ }^{1}$ and Pei-Jen $\mathrm{Lou}^{4}$ \\ ${ }^{1}$ Institute of Biomedical Engineering, College of Medicine and College of Engineering, National Taiwan University, Taipei 10002, Taiwan \\ ${ }^{2}$ Graduate Institute of Biomedical Engineering, National Chung Hsing University, Taichung 40254, Taiwan \\ ${ }^{3}$ Institute of Atomic and Molecular Sciences, Academia Sinica, Taipei 10617, Taiwan \\ ${ }^{4}$ Department of Otolaryngology, National Taiwan University Hospital and College of Medicine, Taipei 10002, Taiwan
}

Correspondence should be addressed to Pei-Jen Lou; pjlou@ntu.edu.tw

Received 21 July 2012; Accepted 6 September 2012

Academic Editor: Tobias Kiesslich

Copyright (C) 2013 Ya-Shuan Chou et al. This is an open access article distributed under the Creative Commons Attribution License, which permits unrestricted use, distribution, and reproduction in any medium, provided the original work is properly cited.

\begin{abstract}
We have applied a fluorescent molecule 3,6-bis(1-methyl-4-vinylpyridinium) carbazole diiodide (BMVC) for tumor targeting and treatment. In this study, we investigated the photo-induced antitumor effect of BMVC. In vitro cell line studies showed that BMVC significantly killed TC-1 tumor cells at light dose greater than $40 \mathrm{~J} / \mathrm{cm}^{2}$. The fluorescence of BMVC in the tumor peaked at 3 hours and then gradually decreased to reach the control level after 24 hours. In vivo tumor treatment studies showed BMVC plus light irradiation (iPDT) significantly inhibited the tumor growth. At day 24 after tumor implantation, tumor volume was measured to be $225 \pm 79 \mathrm{~mm}^{3}, 2542 \pm 181 \mathrm{~mm}^{3}, 1533 \pm 766 \mathrm{~mm}^{3}$, and $1317 \pm 108 \mathrm{~mm}^{3}$ in the iPDT, control, light-only, and BMVC-only groups, respectively. Immunohistochemistry studies showed the microvascular density was significantly lower in the iPDT group. Taken together, our results demonstrated that BMVC may be a potent tumor-specific photosensitizer (PS) for PDT.
\end{abstract}

\section{Introduction}

Tumor-targeting therapy has emerged as an effective and attractive treatment for cancer. Among the various cancerspecific targets tested, telomerase has gathered much attention in recent years. Telomerase is detected in about $85 \%$ to $90 \%$ of cancer cells, but in a low level of normal cells [1]. The maintenance of telomere length by telomerase is required for unlimited proliferation of cancer cells. Telomere has been the target for the development of cancer probes, and telomerase inhibitors have been developed to inhibit telomerase activity and limiting cancer cell growth [2].

In the search for tumor-targeting agents, we have recently developed a fluorescent molecule 3,6-bis(1-methyl-4vinylpyridinium) carbazole diiodide (BMVC) for recognizing specific quadruplex structures such as the $\mathrm{T}_{2} \mathrm{AG}_{3}$ telomeric repeats and inhibiting the telomerase activity [3-5]. Intriguingly, the fluorescence of BMVC detected in cancer cells was much stronger than that in normal cells, suggesting it to be a good candidate for a tumor-targeting agent [3]. The maximum absorption of BMVC is shifted from 435 to $460 \mathrm{~nm}$ and the fluorescence intensity increases significantly when BMVC interacts with DNA [5]. Because of the ability of telomerase inhibition, BMVC induces accelerated senescence of cancer cells [6].

Photodynamic therapy (PDT) is an effective treatment for cancerous and precancerous lesions [7]. The advantages of PDT are that it can be repeated in the same site if necessary, and it is less destructive than traditional surgery. PDT requires PSs that are activated by specific wavelengths of light. Illumination of tumor results in the destruction of cells due to a photochemical reaction. Reactive oxygen species, including singlet oxygen and free radicals, are generated by the photochemical reaction $[8,9]$. This photochemical reaction is capable of inducing cellular apoptosis and necrosis, by evoking oxidative stress [10]. In addition, PDT may cause tumor cell death indirectly by damaging tumor-associated vasculature or activating host immune responses $[9,11]$. 


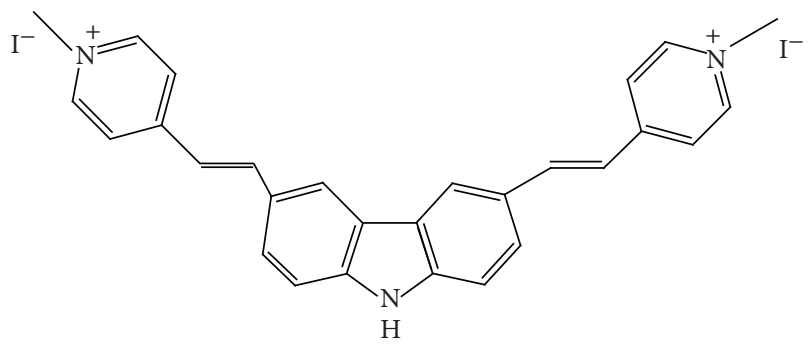

(a)

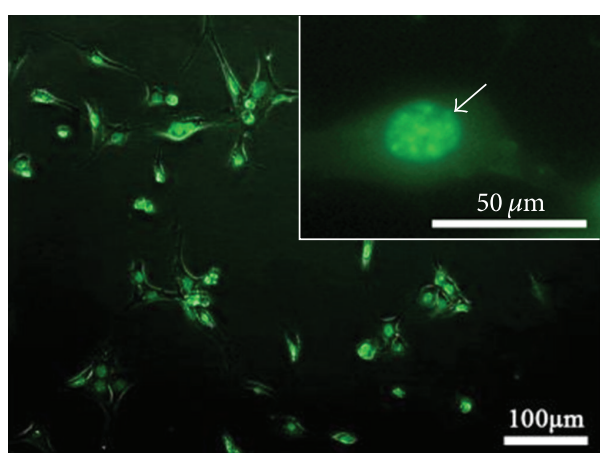

(b)

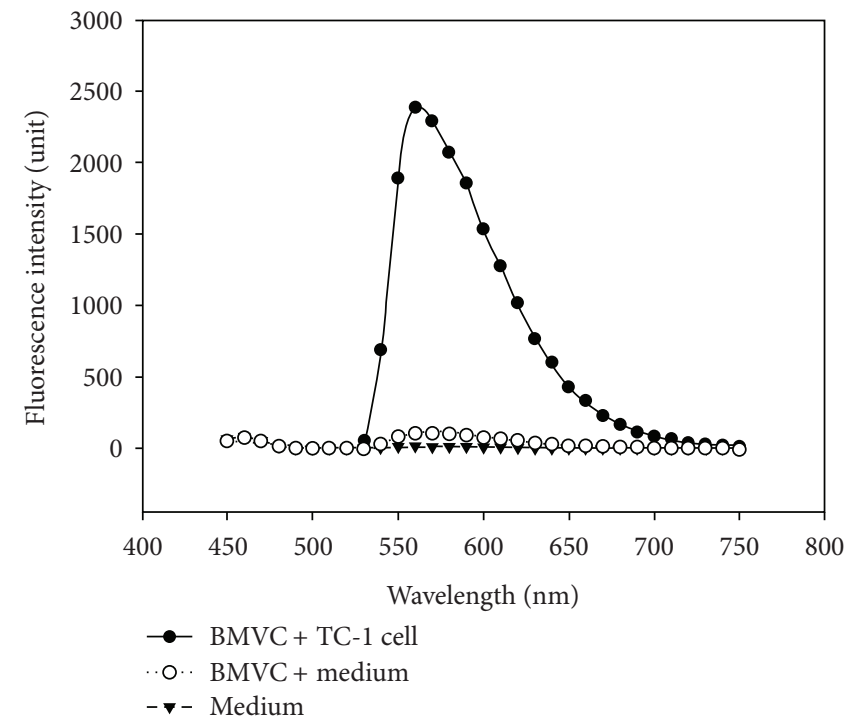

(c)

FIGURE 1: (a) Chemical structure of BMVC. (b) Fluorescence image of TC-1 cells incubated with $20 \mu \mathrm{M}$ of BMVC. (c) Fluorescence spectra of BMVC before and after incubation with TC-1 cells.

Previously we have investigated the fluorescence resonance energy transfer (FRET) binary system that consists of BMVC conjugating porphyrin [12]. We found that PDT efficiency is greater when excited by $470 \mathrm{~nm}$ of light as compared to $510 \mathrm{~nm}$ of light. It is surprised that better PDT efficiency is observed upon exciting the FRET donor (BMVC) rather than the acceptor (porphyrin). This extra phototoxic effect could result from a type I photodynamic reaction [13-15] because we detected neither the characteristic spectral signal of singlet oxygen $(1270 \mathrm{~nm})$ from BMVC in $\mathrm{D}_{2} \mathrm{O}$ solution nor the decrease of 3-diphenylisobenzofuran (DPBF) signal in organic solvent. Here, we have examined the phototoxicity mechanism of BMVC and illustrated its potential to be used as a photosensitizer (PS) for photodynamic therapy (PDT).

Despite the potential advantages in clinical application, PDT has several limitations that hinder its wide clinical acceptance. Among them, sustained skin photosensitivity and low tumor selectivity are two major problems for the PSs [16]. The purpose of this study was to investigate the photochemical effects of BMVC on tumor cells. Cellular cytotoxicity of BMVC was evaluated in TC-1 cell line.
The antitumor effect of BMVC combined with a specific wavelength of light was investigated in the animal model.

\section{Materials and Methods}

BMVC was synthesized from 3,6-dibromocarbazole as described previously [17].

2.1. Cell Line. The mouse (C57BL/6, B6) lung tumor line TC- 1 was maintained in a humidified $5 \% \mathrm{CO}_{2}$ incubator at $37^{\circ} \mathrm{C}$. TC-1 cells were grown in RPMI 1640 supplemented with $10 \%$ fetal calf serum (FCS), $50 \mathrm{U} / \mathrm{mL}$ penicillin, $50 \mathrm{U} / \mathrm{mL}$ streptomycin, and $0.4 \mathrm{mg} / \mathrm{mL}$ G418 [18].

2.2. Fluorescence Spectrum. Cells were treated with $20 \mu \mathrm{M}$ BMVC and then washed, trypsined, and resuspended. The cell suspensions were collected and measured using a fluorescence microplate reader (SpectraMax M2e, Molecular Devices, Sunnyvale, CA, USA) with $460 \mathrm{~nm}$ excitation wavelengths. Fluorescence emission wavelengths of BMVC at 


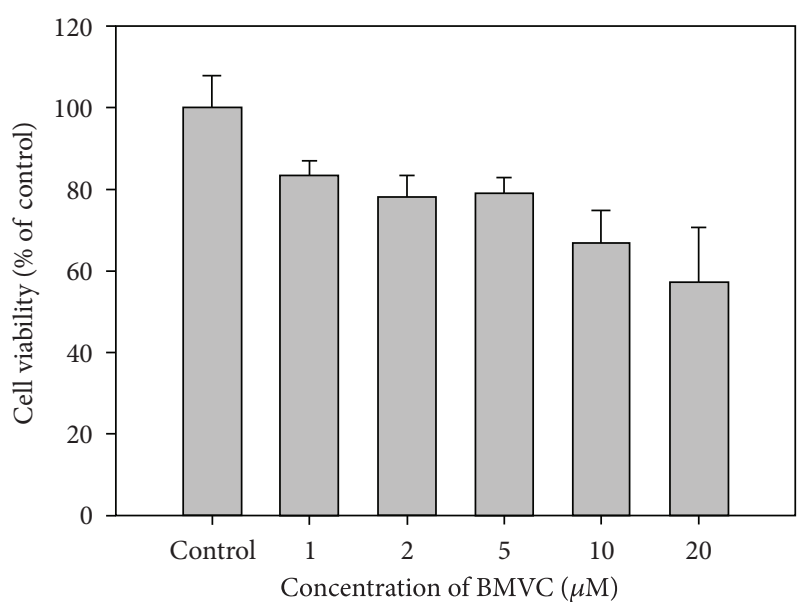

Figure 2: Cytotoxicity of BMVC on TC-1 cells. TC-1 cells were treated with different concentrations of BMVC for 24 hours and cell viability was determined by the Alamar Blue assay.

500-750 nm were recorded by scanning $10 \mathrm{~nm}$ wavelength windows for each sample [19].

2.3. Cytotoxicity Assay. Cells were grown in 96-well plates (2000 cells/well) and then incubated with BMVC for 6 hours and changed to fresh culture medium (without phenol red), and the cytotoxicity was determined by the Alamar Blue assay [20]. 10\% Alamar Blue in $200 \mu \mathrm{L}$ of culture media was added to each test well and analyzed spectrophotometrically at the absorbance difference between 570 and $600 \mathrm{~nm}$.

2.4. Free Radical Assay. The light source for measurement of PDT effect was a white light (a $200 \mathrm{~W}$ Xenon lamp that passes through a $400-700 \mathrm{~nm}$ mirror module) finally, the light power was $100 \mathrm{~mW} / \mathrm{cm}^{-2}$ on the sample surface.

2.5. Animal Study. All animal studies were approved by the Institutional Animal Care Committee. Male C57BL/6 (B6) mice, weighing $20-28 \mathrm{~g}$, were supplied by the University Laboratory Animal Center and were allowed free access to food and water. Around $5 \times 10^{4} \mathrm{TC}-1$ cells in $100 \mu \mathrm{L}$ of HBSS were injected s.c. into B6 mice. BMVC at concentration of $5 \mathrm{mg} / \mathrm{kg}$ was injected (single dose) i.p. after the tumor grew to around 50 to $100 \mathrm{~mm}^{3}$. The length $(L)$ and width $(W)$ of the TC-1 tumor mass and the body weight of mice at 1,7 , 10 , and 14 days after BMVC injection were recorded. Tumors were measured in two orthogonal directions, and the tumor volumes were estimated as $\left(L W^{2}\right) / 2[18,21]$. All animals challenged with TC-1 cells had developed a palpable tumor at day 10 .

2.6. Distribution of BMVC in Mice. Mice were sacrificed at $1,2,3,5,12,24$, and 72 hours after BMVC injection, and the tissues were removed, homogenized, and analyzed by a fluorescence microplate reader (excitation wavelength at $460 \mathrm{~nm}$ ). Tissues from mice 3 hours after BMVC injection were collected, washed with PBS, and then analyzed by the

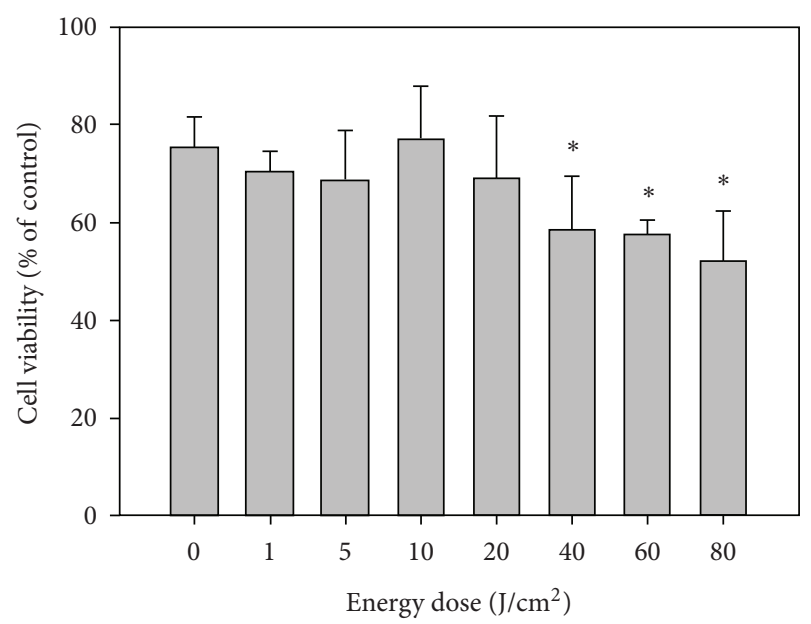

FIgURE 3: Photodynamic effect of BMVC. TC-1 cells were incubated with BMVC and then subjected to light treatment as described in Section 2. Cell viability was measured. ${ }^{*} P<0.05$ as compared to the $0 \mathrm{~J} / \mathrm{cm}^{2}$.

flow cytometry (FACS Calibur and CellQuest software, BD Biosciences).

\subsection{Photodynamic Therapy}

2.7.1. In Vitro Study. Cells were incubated with $5 \mu \mathrm{M}$ BMVC in darkness for 6 hours at $37^{\circ} \mathrm{C}$. After wash with PBS, the cells were immediately exposed to different doses of light at $445 \mathrm{~nm}$ (LDM445, Pkebtunax, Germany). Cell toxicity was determined 24 hours after light treatment with energy doses of $1,5,10,20,40,60$, and $80 \mathrm{~J} / \mathrm{cm}^{2}$.

2.7.2. In Vivo Study. BMVC $(5 \mathrm{mg} / \mathrm{kg})$ was injected to the mice when the size of tumor reached $0.8 \mathrm{~cm}$ in diameter (about 14 days after TC-1 injection). Three hours after BMVC injection, mice were subjected to interstitial light treatment as described before $[22,23]$. In brief, a needle $(21 \mathrm{G})$ was pushed percutaneously into the tumor, and the fiber was passed through the needle. The tip of the fiber was managed to be at about $1 \mathrm{~mm}$ outside of the needle tip to ensure it actually touched the tumor tissue. A single dose of $150 \mathrm{~J}$ was delivered to the tumor by a $445 \mathrm{~nm}$ diode laser (LDM445, Pkebtunax, Germany).

2.8. Immunohistochemistry. Cryosections of the tumor at $10 \mu \mathrm{m}$ thickness were fixed with $10 \%$ formalin, washed, and then immunostained for $\alpha$-smooth muscle actin ( $\alpha$-SMA, $2 \mu \mathrm{g} / \mathrm{mL}$, DAKO, Glostrup, Denmark). The number of $\alpha$-SMA positive cells was counted in three nonoverlapping regions. Unit counts were expressed as the number of $\alpha$-SMA positive unit per $\mathrm{mm}^{2}$ of tumor tissue.

2.9. Statistical Analysis. Student's $t$-test was used to evaluate the response to a change in conditions. Data were subjected to statistical analysis using the SPSS for Windows version 10 . 


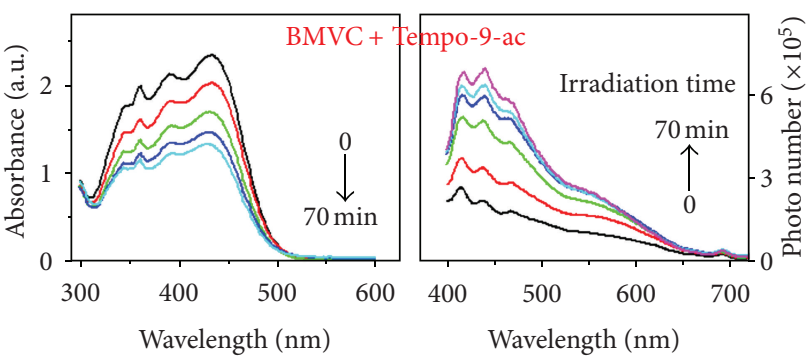

(a)

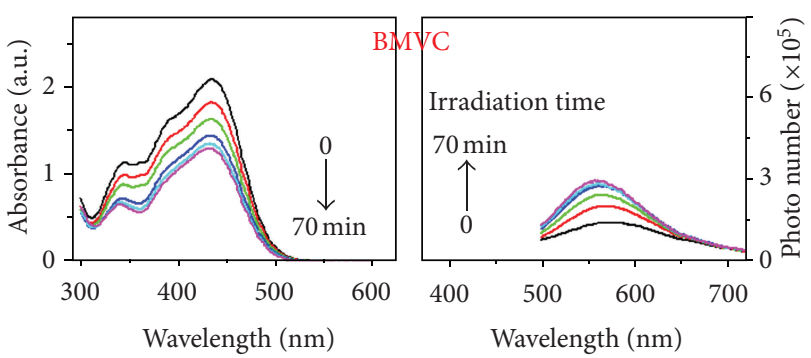

(b)

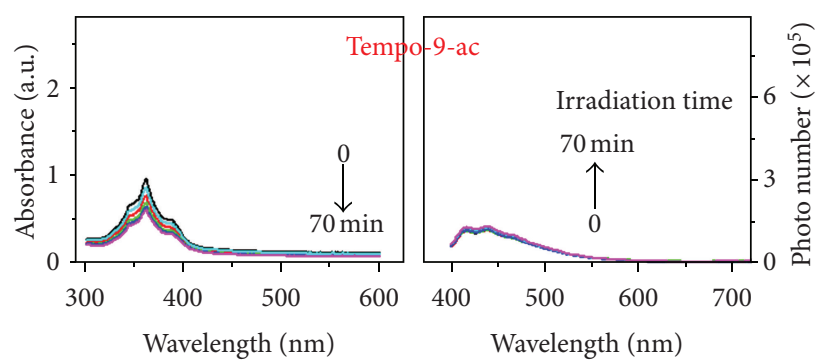

(c)

FIGURE 4: Absorption (left) and emission (right) spectra BMVC upon irradiation (400-700 $\mathrm{nm} 100 \mathrm{~mW} / \mathrm{cm}^{2}$ ) with (a) and without (b) radical fluorescence probe TEMPO-9-ac. (c) Absorption and emission of Tempo-9-ac under similar irradiation condition. The excitation wavelength for emission spectra: $360 \mathrm{~nm}$ for (a) and (c), $440 \mathrm{~nm}$ for (b).

\section{Results}

3.1. BMVC Fluorescence Spectra. The chemical structure of BMVC was shown in Figure 1(a). When TC-1 cells were cultured in the presence of BMVC, the fluorescence signals were detected mainly in the cell nuclei (Figure 1(b)). Fluorescence emission spectra of BMVC were depicted in Figure 1(c). It clearly demonstrated that the fluorescence intensity of BMVC increased for almost 2 orders of magnitude upon interacting with cells.

3.2. In Vitro Cytotoxicity and Phototoxicity. Cytotoxic effects of BMVC to TC-1 cells were evaluated. Low dose BMVC treatment did not have apparent toxicity to the cells. As shown in the Figure 2, $\sim 80 \%$ of cells were still viable when the BMVC concentrations were below $5 \mu \mathrm{M}$. When the BMVC concentrations were above this level, cytotoxicity effects became obvious. The cell viability decreased to $\sim 60 \%$ when cells were treated with $20 \mu \mathrm{M}$ of BMVC. Since $5 \mu \mathrm{M}$ of BMVC treatment did not show significant cytotoxicity to the cells, we use this concentration for the subsequent PDT treatment.

Photo-induced cytotoxicity of BMVC was evaluated as a function of light dose. Figure 3 showed the photo-induced cytotoxicity of BMVC upon the treatment of $0,1,5,10,20$, 40,60 , and $80 \mathrm{~J} / \mathrm{cm}^{2}$ light doses in TC- 1 cells. It was found that BMVC coupled with low light doses (below $20 \mathrm{~J} / \mathrm{cm}^{2}$ ) did not show apparent cytotoxicity. However, cell survival of the BMVC coupled with higher light doses $(40,60$, and $80 \mathrm{~J} / \mathrm{cm}^{2}$ ) appreciably decreased to $66.9 \%, 57.5 \%$, and $52.1 \%$, respectively.

Type I sensitization assumes the formation of radicals via electron transfer (reductive or oxidative) involving the triplet state of the photosensitizer. The free radical probe 4((9-acridinecarbonyl) amino)-2,2,6,6-tetramethylpiperidin1-oxyl (TEMPO-9-ac, Invitrogen, Carlsbad, CA, USA) captures radicals (mostly long-lived carbon or sulfur centered) resulting in fluorescence turnon $(\lambda \mathrm{ex} / \lambda \mathrm{em}=360 / 440 \mathrm{~nm})$ [24]. Irradiation of BMVC $(10 \mu \mathrm{M})$ generated apparent increase in signal from TEMPO-9-ac $(10 \mu \mathrm{M})$ (Figures $4(\mathrm{a})$ and 4(b)), compared to unchanged levels of the control. (Figure 4(c)). As the most type I cases [25, 26], Figure 4(a) also showed light-induced photo reduction of the BMVC, which was accompanied by bleaching, resulting in the longrange electron transfer through the water channel, followed by the superoxide generation [27]. This is why we cannot collect similar results as Figure 4 in DMSO solution (data not shown).

3.3. Distribution and Kinetics of BMVC in the Tumor and Normal Mice Tissues. Liver and kidney are the main organs responsible for drug metabolism and excretion. We further measured the distribution of BMVC in the tumor, liver, and kidney tissues by flow cytometry and fluorescence microscopy at 3 hours after BMVC injection (Figure 5). The results showed that the majority $(90.8 \%)$ of tumor cells were positive for BMVC fluorescence. On the other hand, only $47.2 \%$ of the liver cells and $30.9 \%$ of the kidney cells were positive for BMVC fluorescence, although the fluorescence intensity was stronger in liver and kidney than in the tumor. Kinetics of BMVC in the tumor and normal mice tissues were also investigated. After injection, the fluorescence of BMVC gradually increased in the tumor tissues, peaked at 3 hours, and then gradually decreased to reach the control level after 24 hours (Figure 6(a)). Similarly, the fluorescence of BMVC 

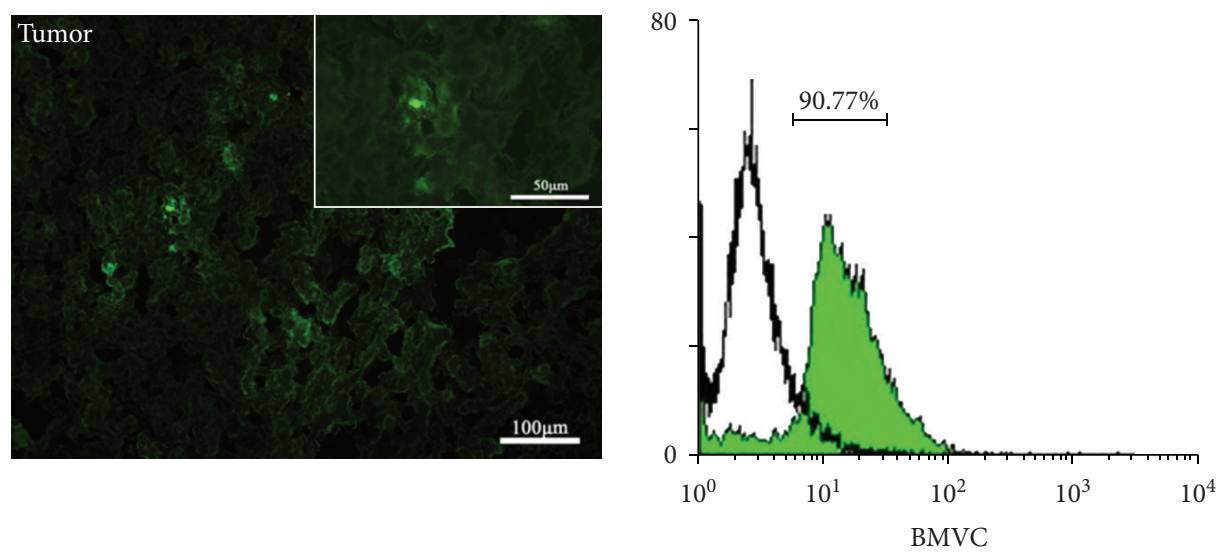

(a)
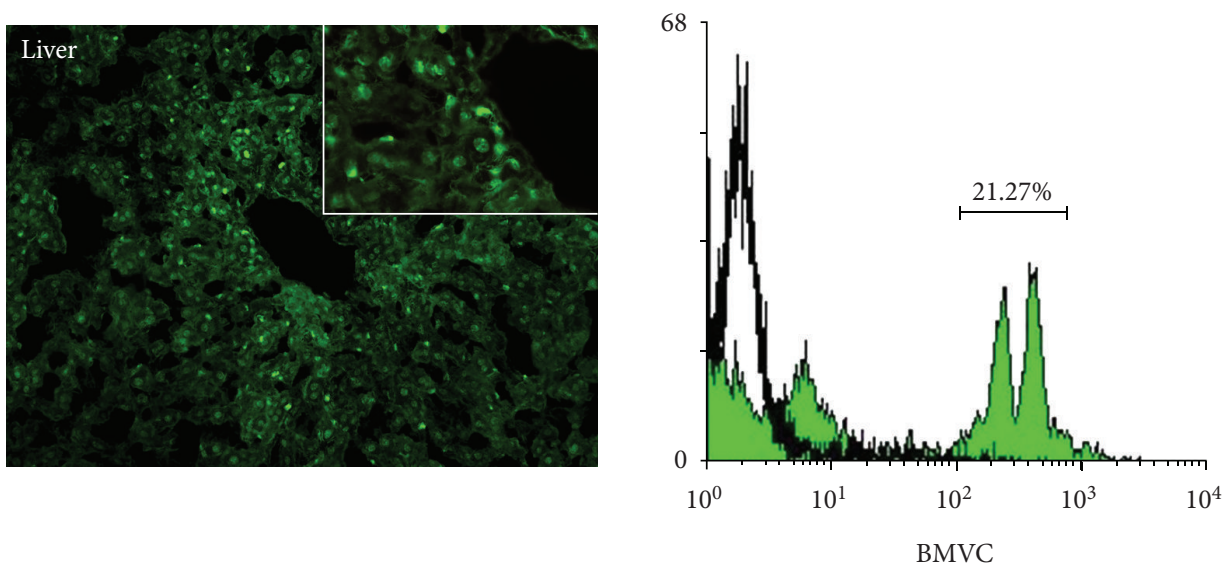

(b)
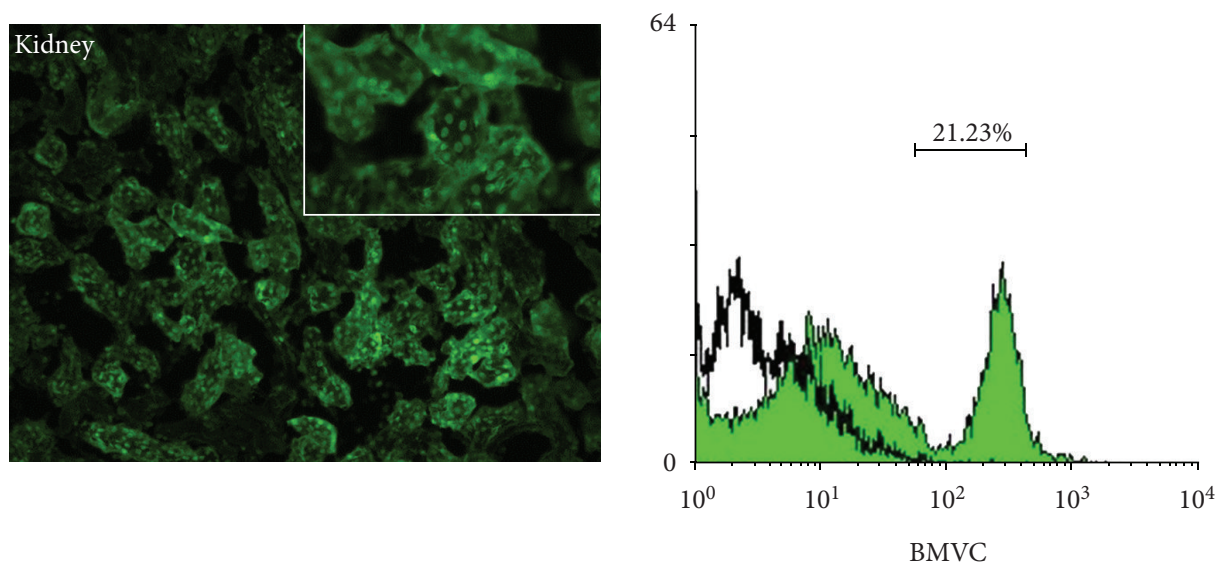

(c)

FIGURE 5: Distribution of BMVC in the tumor and normal mice tissues. Flow cytometry (left panel) and fluorescence images (right panel) of tumor (a), liver (b), and kidney (c) tissues were shown. The numbers in percentage shown in the left panel indicated the ratio of BMVC positive cells to the total number of cells.

in the liver and kidney increased after drug injection, peaked at 24 and 12 hours, respectively, and then gradually decreased (Figures 6(b) and 6(c)). At 72 hours after injection, the BMVC fluorescence levels in the liver and kidney were still above the control level. However, the BMVC fluorescence in the brain, lung, and muscles showed no appreciable difference from the control tissues (data not shown).
3.4. In Vivo Tumoricidal Effects. To evaluate the in vivo acute toxicity of BMVC in tumor-bearing mice, we injected TC1 cells s.c. to $\mathrm{B} 6$ mice. After tumors grew to about $50 \mathrm{~mm}^{3}$, mice received i.v. injection of $5 \mathrm{mg} / \mathrm{kg}$ BMVC once. We then evaluated the effect of BMVC on tumor growth and body weight. The results showed no change in body weight between groups (data not shown), and there was no sign 


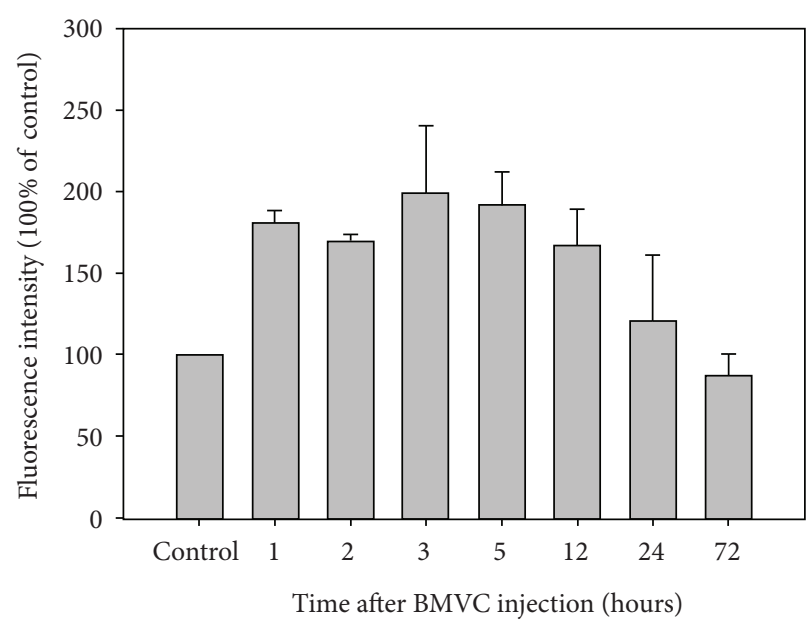

(a)

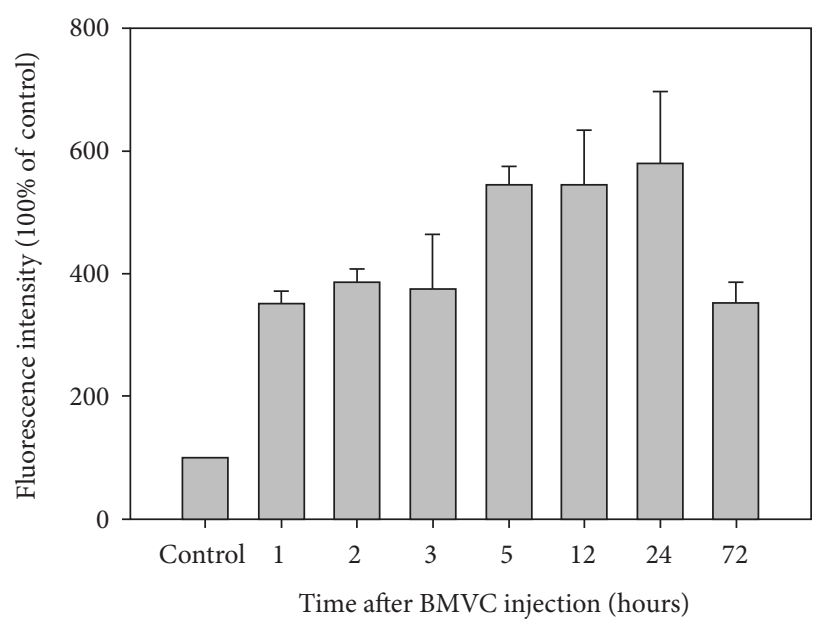

(b)

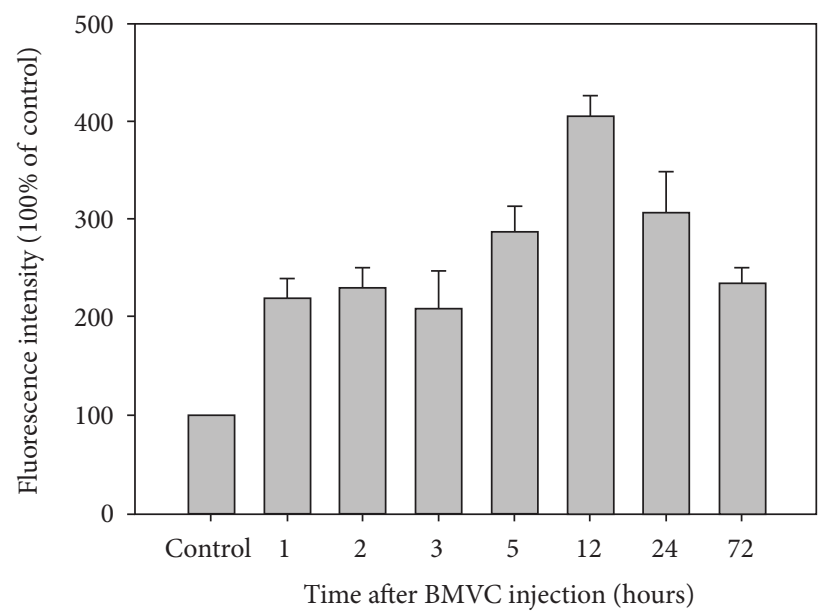

(c)

Figure 6: Kinetic of BMVC in the tumor and normal mice tissues. Mice were treated with BMVC and then sacrificed at different time points as described in Section 2. Fluorescence intensity of BMVC in the tumor (a), liver (b), and kidney (c) tissues was determined by the ELISA reader.

of acute toxicity in this BMVC dose. Figure 7 showed the growth curves of tumors under different treatments. It clearly showed that BMVC plus light (iPDT) significantly inhibited the tumor growth in vivo $(P<0.05)$. At day 24 after tumor implantation, tumor volume was measured to be $225 \pm$ $79 \mathrm{~mm}^{3}, 2542 \pm 181 \mathrm{~mm}^{3}, 1533 \pm 766 \mathrm{~mm}^{3}$, and $1317 \pm$ $108 \mathrm{~mm}^{3}$, respectively, in the iPDT, control, light-only, and BMVC-only groups. There is only $6.76 \%$ evolution of tumor volume in iPDT group between day 14 and 24. Although the tumors in the light-only and the BMVC-only groups were also smaller than the control group, the difference did not reach statistically significant level.

3.5. Immunohistochemistry. PDT is known to cause microvascular destruction in tumor tissues. The direct cytotoxic activity and microvascular damage contribute to the destruction of tumor $[9,28]$. To investigate the microvascular density in the tumors, we measured $\alpha$-smooth muscle actin ( $\alpha$-SMA) positive cells in different groups of tumors. Figure 8 showed the quantification of $\alpha$-SMA positive cells under different conditions. It is found that the microvascular density was significantly lower after both the light-only and the iPDT treatments. On the other hand, BMVC treatment showed minor effect on the microvascular density of tumors.

\section{Discussion}

PDT has emerged as an effective means for cancer therapy. PSs are an essential element of PDT. In general, the photoactivation of the PS leads to an oxygen dependent oxidativereaction resulting in cellular photodamage. The mechanisms of PDT involve direct oxidation of biological targets through hydrogen abstraction or electron transfer to yield radical chain reaction (type I reaction), as well as oxidation mediated by singlet oxygen $\left({ }^{1} \mathrm{O}_{2}\right)$ through energy transfer from triplets to molecular oxygen to initiate oxidative damage (type II reaction). In this study, we investigated the possibility of using BMVC as a PS for PDT. Illuminating TC-1 cells with 


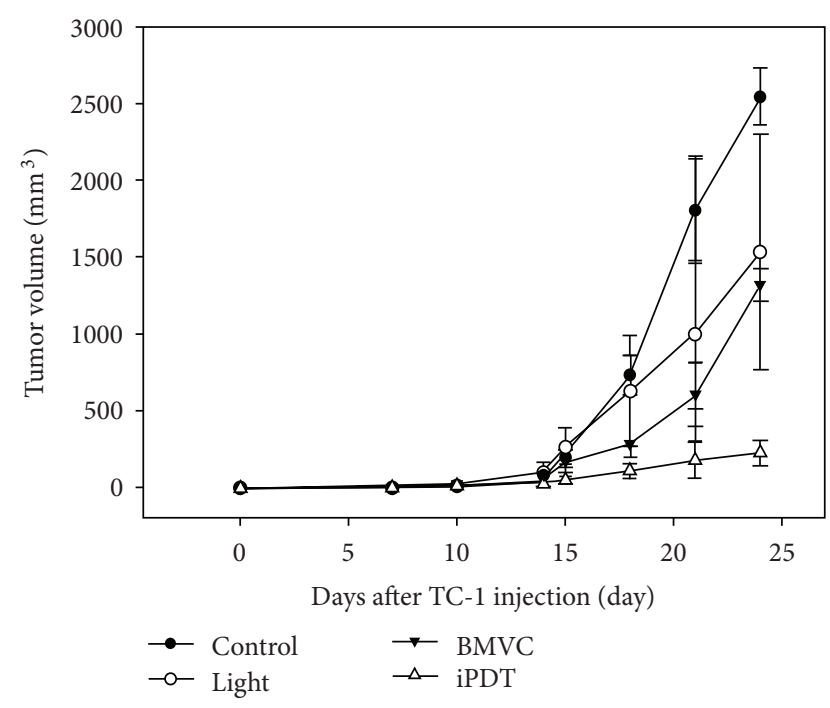

(a)
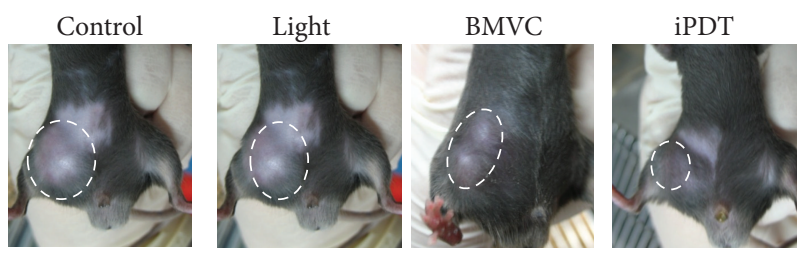

(b)

FIgURE 7: Antitumor effects of BMVC. Mice were transplanted with TC-1 cells and then subjected to light-alone, BMVC-alone, or BMVC plus light (iPDT) treatment as indicated. The changes in tumor volume (a) and pictures of tumors at 24 days posttumor transplantation (b) were shown. Arrow indicates the time point of treatment.

$445 \mathrm{~nm}$ of light can result in the destruction of cells (Figures 2 and 3). Further experiments showed that BMVC plus light (iPDT) significantly inhibited the growth of tumor cells in vivo (Figure 7). These results suggest that BMVC is a useful PS for PDT.

Tumor-targeting therapy is the treatment of cancer cells without injuring the normal cells. However, one of the major drawbacks of current PSs is the lack of tumor selectivity. Many studies have been focused on the modification of specific porphyrin structures to achieve better tumor selectivity. Furthermore, the fluorescence of BMVC detected in cancer cells was much stronger than that in normal cells, letting it to be a good candidate for the tumor-targeting agent [3]. In this study, we showed that $90.8 \%$ of tumor cells were positive for BMVC fluorescence at 3 hours after i.v. injection (Figure 5(a)). On the other hand, only $47.2 \%$ of the liver cells and $30.9 \%$ of the kidney cells were positive for BMVC fluorescence (Figures 5(b) and 5(c)). Together, these results indicated that BMVC had certain degree of tumor selectivity and could be implemented as a tumor-targeting PS for PDT.

In addition to be used in the treatment of cancer, PSs were also implemented in the diagnosis of cancers. In 1924, red fluorescence of porphyrin was observed in experimental rat sarcomas by Policard [29]. This is the first observation of PS fluorescence in tumors. The followed experiments confirmed that some PSs accumulated in cancer cells and were possibly been used in cancer detection (photodynamic diagnosis, PDD) [30]. In PDD, fluorescence in cancer cells could be observed by either fluorescence spectroscopy or fluorescence imaging. Clinical application of PDD had been shown in premalignant oral tissue screening [31]. In this study, we noted that the fluorescence intensity of BMVC increased significantly upon interacting with tumor cells (Figure 1(c)). The emission spectrum was different between free BMVC molecules and BMVC plus TC-1 cells. These results were in line with our previous molecular chemical studies that showed strong fluorescence of BMVC that peaked at approximately 550 or $575 \mathrm{~nm}$ in the presence of DNA structure [5]. Since the fluorescence of BMVC significantly enhanced upon interacting with tumor cells and that $90.8 \%$ of in vivo tumor cells exhibited strong BMVC fluorescence, BMVC could be used as a potential molecule for PDD of cancer cells.

Although BMVC-PDT is effective in vitro, there are some limitations in the in vivo animal studies. The absorption wavelength ( $445 \mathrm{~nm}$ ) of BMVC is insufficient to pass through the whole bulk of tumor cells. To overcome this problem, we used fine-needle interstitial light irradiation to shine the light into tumor tissues. This fine-needle interstitial PDT (iPDT) which combines with the BMVC treatment significantly inhibited tumor growth (Figure 7). There were 2 possible mechanisms to explain this significant tumor inhibition. One is the direct effect of BMVC on the tumor telomeres. As reported in our previous studies, BMVC was able to suppress the telomerase activity and induce senescence of cancer cells [3, 4]. Tumor formation and progression can be suppressed by BMVC treatment alone. The other is the effect of PDT may destruct the intratumoral vasculature and result in tumor ischemia and death by decreases in perfusion [21]. Our immunohistochemistry results in this study confirmed the destruction of intratumoral vasculature by BMVC-PDT (Figure 8). Intriguingly, tumors receiving light irradiation alone also showed significant reduction of intratumoral microvascular density. The thermal effect might decrease the vascular density in light irradiation alone group because we treated the mice by a high energy direct treatment. The mild thermal therapy has the ability to change vascular perfusion and oxygen supplied within the tumor microenvironment [32]. Nevertheless, the iPDT group has the smallest tumor volume certainly. In our study, we showed the photochemical effect occurred by BMVC treated combined with light. The mechanism behind this light induced microvascular destruction remains elusive and needs further investigation.

\section{Conclusions}

We described a tumor-targeting therapy for the application of BMVC. In summary, the distinct properties of this fluorescent molecule provide a design of PS for PDT treatment. PDT experiments showed that BMVC-PDT significantly inhibited 

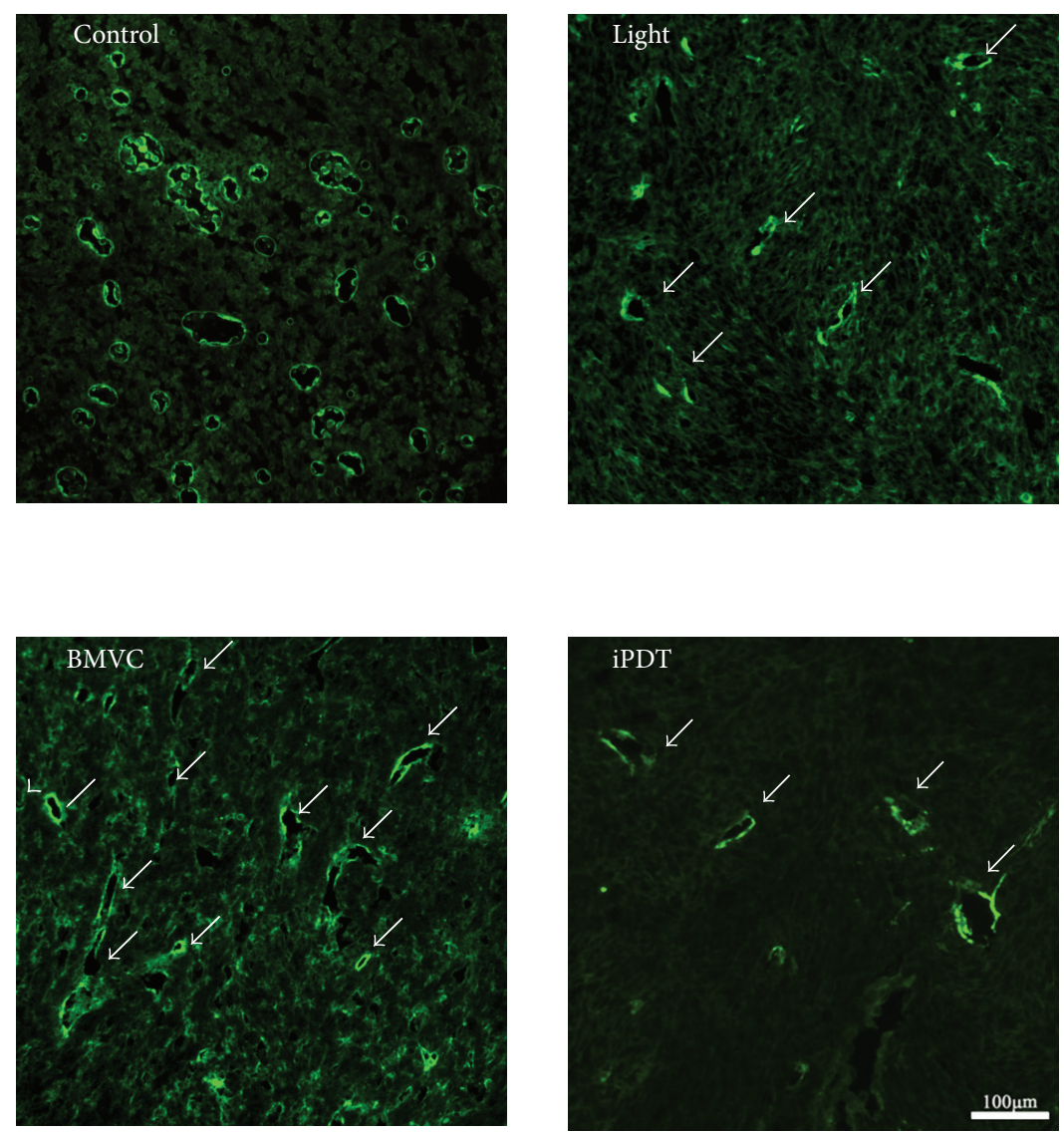

(a)

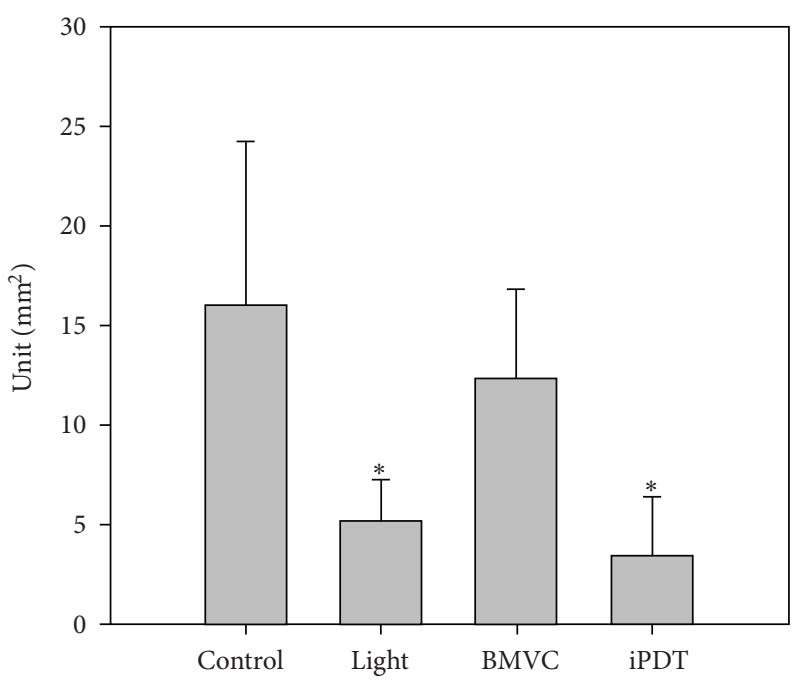

(b)

FIGURE 8: Microvascular densities of control and iPDT treated tumors. Immunohistochemical staining for $\alpha$-SMA (a) and semiquantitative analyses results (b) were shown. ${ }^{*} P<0.05$. 
the growth of tumor cells both in the in vitro and in vivo studies. Our results demonstrated that BMVC may be a potent tumor-specific PS for PDT. A number of potential refinements can be incorporated into future studies based on this effect of BMVC, such as conjugate with other PSs to increase phototoxic effects and pi-conjugation lengths, which induce the bathochromic shift to enhance the penetration depth of the PDT laser.

\section{Abbreviations}

\begin{tabular}{|c|c|}
\hline BMVC: & $\begin{array}{l}\text { 3,6-Bis (1-methyl-4-vinylpyridinium) } \\
\text { carbazole diiodide }\end{array}$ \\
\hline DPBF: & 3-Diphenylisobenzofuran \\
\hline FCS: & Fetal calf serum \\
\hline FRET: & Fluorescence resonance energy transfer \\
\hline PDD: & Photodynamic diagnosis \\
\hline PDT: & Photodynamic therapy \\
\hline PS: & Photosensitizer. \\
\hline
\end{tabular}

\section{Acknowledgments}

This work was supported financially by the National Science Council (NSC-100-2218-E-001-001) of Taiwan, and the Eighth Core Laboratory and Animal Center of National Taiwan University, Taipei, Taiwan.

\section{References}

[1] J. W. Shay and S. Bacchetti, "A survey of telomerase activity in human cancer," European Journal of Cancer Part A, vol. 33, no. 5, pp. 787-791, 1997.

[2] J. W. Shay and W. E. Wright, "Telomerase: a target for cancer therapeutics," Cancer Cell, vol. 2, no. 4, pp. 257-265, 2002.

[3] C. C. Chang, I. C. Kuo, J. J. Lin et al., "A novel carbazole derivative, BMVC: a potential antitumor agent and fluorescence marker of cancer cells.," Chemistry \& Biodiversity, vol. 1, no. 9, pp. 1377-1384, 2004.

[4] C. C. Chang, I. C. Kuo, I. F. Ling et al., "Detection of quadruplex DNA structures in human telomeres by a fluorescent carbazole derivative," Analytical Chemistry, vol. 76, no. 15, pp. 4490-4494, 2004.

[5] C. C. Chang, J. Y. Wu, C. W. Chien et al., "A fluorescent carbazole derivative: high sensitivity for quadruplex DNA," Analytical Chemistry, vol. 75, no. 22, pp. 6177-6183, 2003.

[6] F. C. Huang, C. C. Chang, P. J. Lou et al., "G-quadruplex stabilizer 3,6-bis(1-methyl-4-vinylpyridinium)carbazole diiodide induces accelerated senescence and inhibits tumorigenic properties in cancer cells," Molecular Cancer Research, vol. 6, no. 6, pp. 955-964, 2008.

[7] R. Bhuvaneswari, Y. Y. Gan, K. C. Soo, and M. Olivo, "The effect of photodynamic therapy on tumor angiogenesis," Cellular and Molecular Life Sciences, vol. 66, no. 14, pp. 2275-2283, 2009.

[8] K. R. Weishaupt, C. J. Gomer, and T. J. Dougherty, "Identification of singlet oxygen as the cytotoxic agent in photo inactivation of a murine tumor," Cancer Research, vol. 36, no. 7, 1976.

[9] T. J. Dougherty, C. J. Gomer, B. W. Henderson et al., "Photodynamic therapy," Journal of the National Cancer Institute, vol. 90, no. 12, pp. 889-905, 1998.
[10] A. D. Garg, D. V. Krysko, P. Vandenabeele, and P. Agostinis, "DAMPs and PDT-mediated photo-oxidative stress: exploring the unknown," Photochemical and Photobiological Sciences, vol. 10, no. 5, pp. 670-680, 2011.

[11] M. C. A. Issa and M. Manela-Azulay, "Photodynamic therapy: a review of the literature and image documentation," Anais Brasileiros de Dermatologia, vol. 85, no. 4, pp. 501-511, 2010.

[12] C. C. Chang, M. C. Hsieh, J. C. Lin, and T. C. Chang, "Selective photodynamic therapy based on aggregation-induced emission enhancement of fluorescent organic nanoparticles," Biomaterials, vol. 33, no. 3, pp. 897-906, 2012.

[13] N. Houba-Herin, C. M. Calberg-Bacq, and A. Van De Vorst, "Photodynamic activity of acridine orange: peroxide radical induction in DNA and synthetic polynucleotides," International Journal of Radiation Biology, vol. 45, no. 5, pp. 487-495, 1984.

[14] C. T. Chu, D. J. Levinthal, S. M. Kulich, E. M. Chalovich, and D. B. DeFranco, "Oxidative neuronal injury: the dark side of ERK1/2," European Journal of Biochemistry, vol. 271, no. 11, pp. 2060-2066, 2004.

[15] R. B. Vegh, K. M. Solntsev, M. K. Kuimova et al., "Reactive oxygen species in photochemistry of the red fluorescent protein "killer Red"', Chemical Communications, vol. 47, no. 17, pp. 4887-4889, 2011.

[16] S. B. Brown, E. A. Brown, and I. Walker, "The present and future role of photodynamic therapy in cancer treatment," Lancet Oncology, vol. 5, no. 8, pp. 497-508, 2004.

[17] C. C. Chang, J. Y. Wu, and T. C. Chang, "A carbazole derivative synthesis for stabilizing the quadruplex structure," Journal of the Chinese Chemical Society, vol. 50, no. 2, pp. 185-188, 2003.

[18] L. G. Bermúdez-Humarán, N. G. Cortes-Perez, F. Lefèvre et al., "A novel mucosal vaccine based on live lactococci expressing E7 antigen and IL-12 induces systemic and mucosal immune responses and protects mice against human papillomavirus type 16-induced tumors," Journal of Immunology, vol. 175, no. 11, pp. 7297-7302, 2005.

[19] T. C. Chang and C. C. Chang, "Detection of G-quadruplexes in cells and investigation of G-quadruplex structure of d(T2AG3)4 in $\mathrm{K}+$ solution by a carbazole derivative: BMVC," Methods in Molecular Biology, vol. 608, pp. 183-206, 2010.

[20] J. O'Brien, I. Wilson, T. Orton, and F. Pognan, "Investigation of the Alamar Blue (resazurin) fluorescent dye for the assessment of mammalian cell cytotoxicity," European Journal of Biochemistry, vol. 267, no. 17, pp. 5421-5426, 2000.

[21] T. M. Busch, H. W. Wang, E. P. Wileyto, G. Yu, and R. M. Bunte, "Increasing damage to tumor blood vessels during motexafin lutetium-pdt through use of low fluence rate," Radiation Research, vol. 174, no. 3, pp. 331-340, 2010.

[22] L. K. Lee, C. Whitehurst, M. L. Pantelides, and J. V. Moore, "An interstitial light assembly for photodynamic therapy in prostatic carcinoma," BJU International, vol. 84, no. 7, pp. 821-826, 1999.

[23] D. I. Fielding, G. A. Buonaccorsi, A. J. MacRobert, A. M. Hanby, M. R. Hetzel, and S. G. Bown, "Fine-needle interstitial photodynamic therapy of the lung parenchyma: photosensitizer distribution and morphologic effects of treatment," Chest, vol. 115, no. 2, pp. 502-510, 1999.

[24] C. Aliaga, A. Aspée, and J. C. Scaiano, "A new method to study antioxidant capability: hydrogen transfer from phenols to a prefluorescent nitroxide," Organic Letters, vol. 5, no. 22, pp. 4145-4148, 2003.

[25] M. E. Bulina, D. M. Chudakov, O. V. Britanova et al., "A genetically encoded photosensitizer," Nature Biotechnology, vol. 24, no. 1, pp. 95-99, 2006. 
[26] M. E. Bulina, K. A. Lukyanov, O. V. Britanova, D. Onichtchouk, S. Lukyanov, and D. M. Chudakov, "Chromophoreassisted light inactivation (CALI) using the phototoxic fluorescent protein KillerRed," Nature Protocols, vol. 1, no. 2, pp. 947-953, 2006.

[27] A. Roy, P. Carpentier, D. Bourgeois, and M. Field, "Diffusion pathways of oxygen species in the phototoxic fluorescent protein KillerRed," Photochemical and Photobiological Sciences, vol. 9, no. 10, pp. 1342-1350, 2010.

[28] V. H. Fingar, T. J. Wieman, and P. S. Haydon, "The effects of thrombocytopenia on vessel stasis and macromolecular leakage after photodynamic therapy using photofrin," Photochemistry and Photobiology, vol. 66, no. 4, pp. 513-517, 1997.

[29] A. Policard, "Etude sur les aspects offerts par des tumeurs experimentalees examinees a la lumikere de Wood," Comptes Rendus des Seances et Memoires de la Societe de Biologie, vol. 91, pp. 1423-1428, 1924.

[30] L. Leive, "A nonspecific increase in permeability in Escherichia coli produced by Edta," Proceedings of the National Academy of Sciences, vol. 53, no. 4, pp. 745-750, 1965.

[31] H. M. Chen, C. P. Chiang, C. You, T. C. Hsiao, and C. Y. Wang, "Time-resolved autofluorescence spectroscopy for classifying normal and premalignant oral tissues," Lasers in Surgery and Medicine, vol. 37, no. 1, pp. 37-45, 2005.

[32] C. T. Lee, T. MacE, and E. A. Repasky, "Hypoxia-driven immunosuppression: a new reason to use thermal therapy in the treatment of cancer?" International Journal of Hyperthermia, vol. 26, no. 3, pp. 232-246, 2010. 

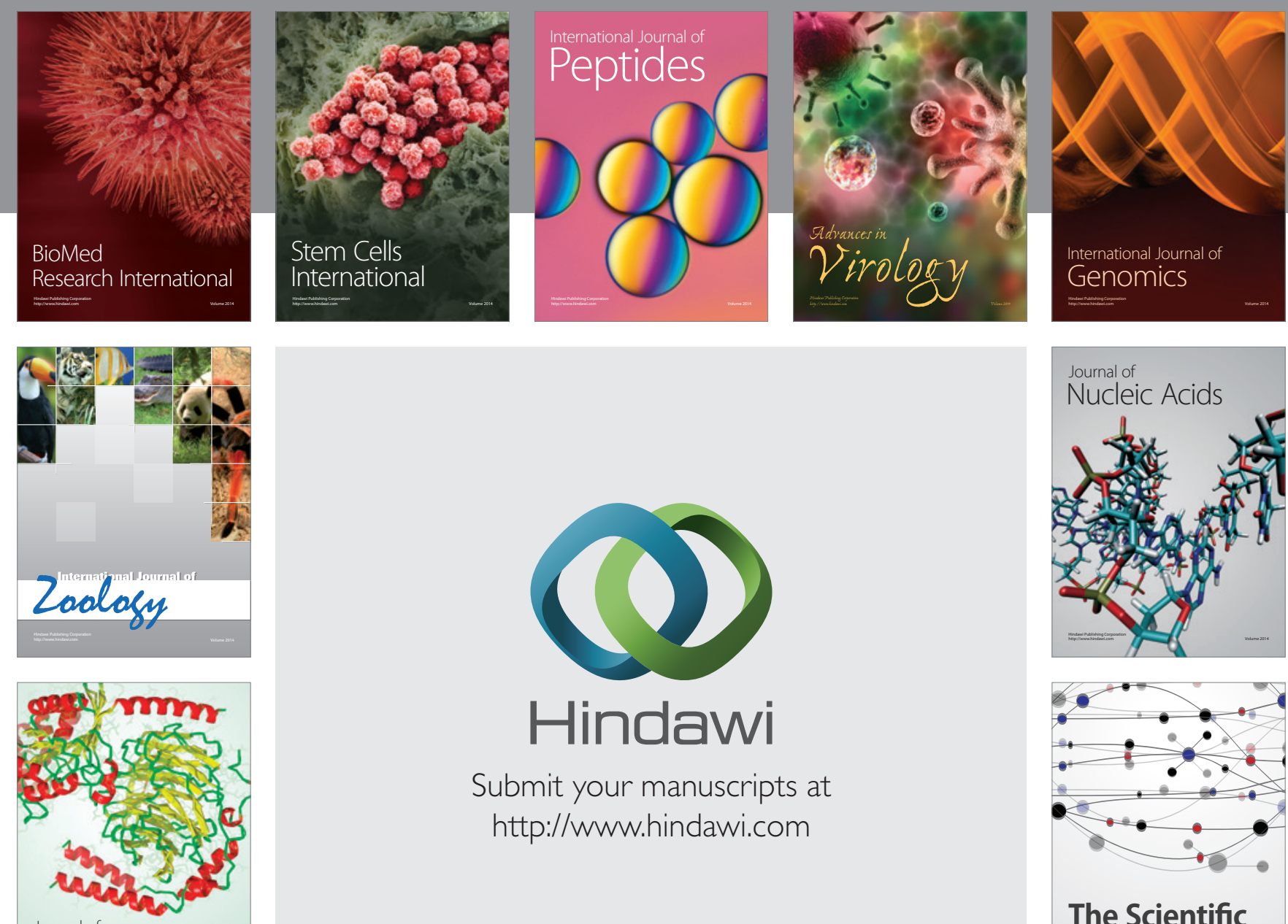

Submit your manuscripts at

http://www.hindawi.com

Journal of
Signal Transduction
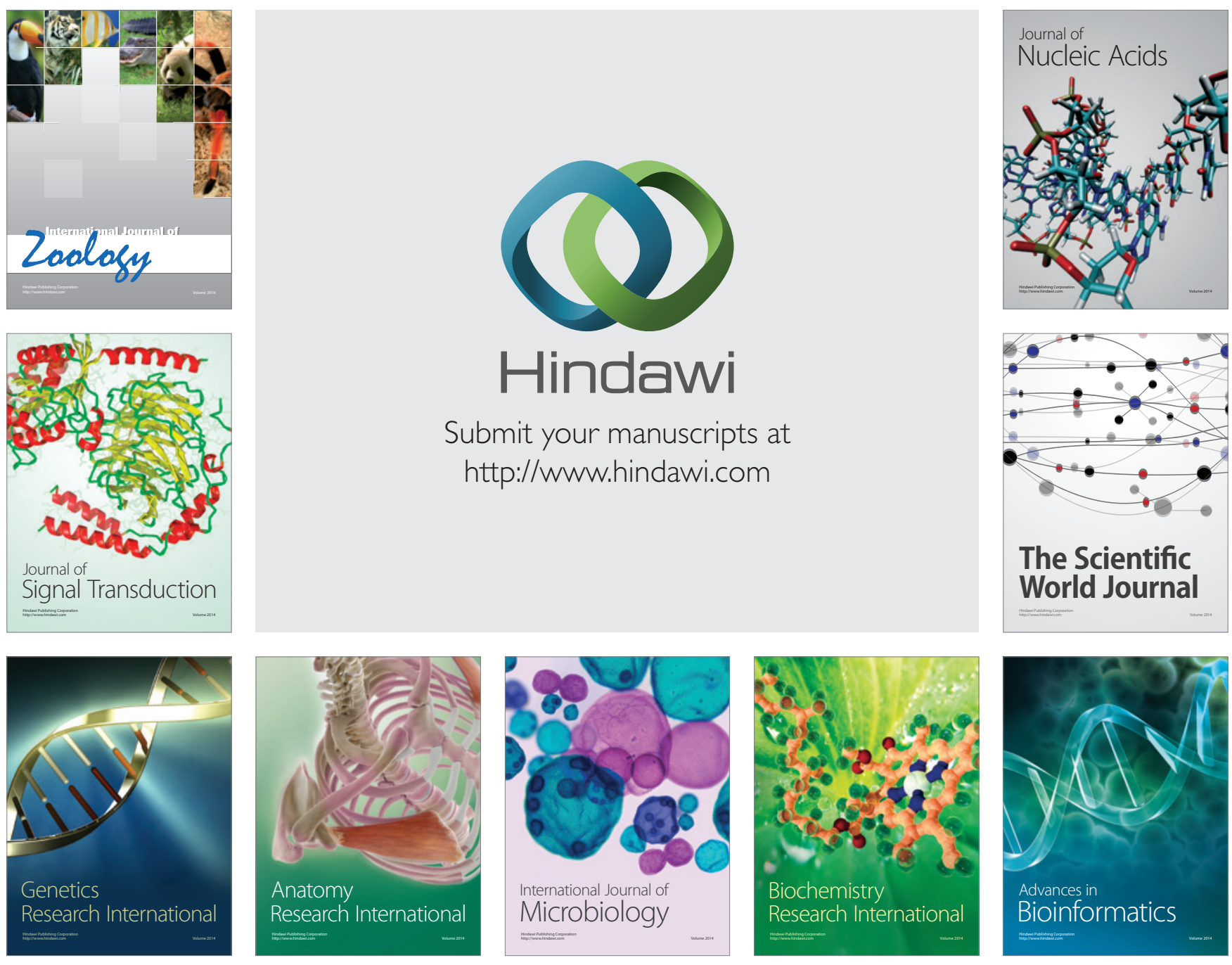

The Scientific World Journal
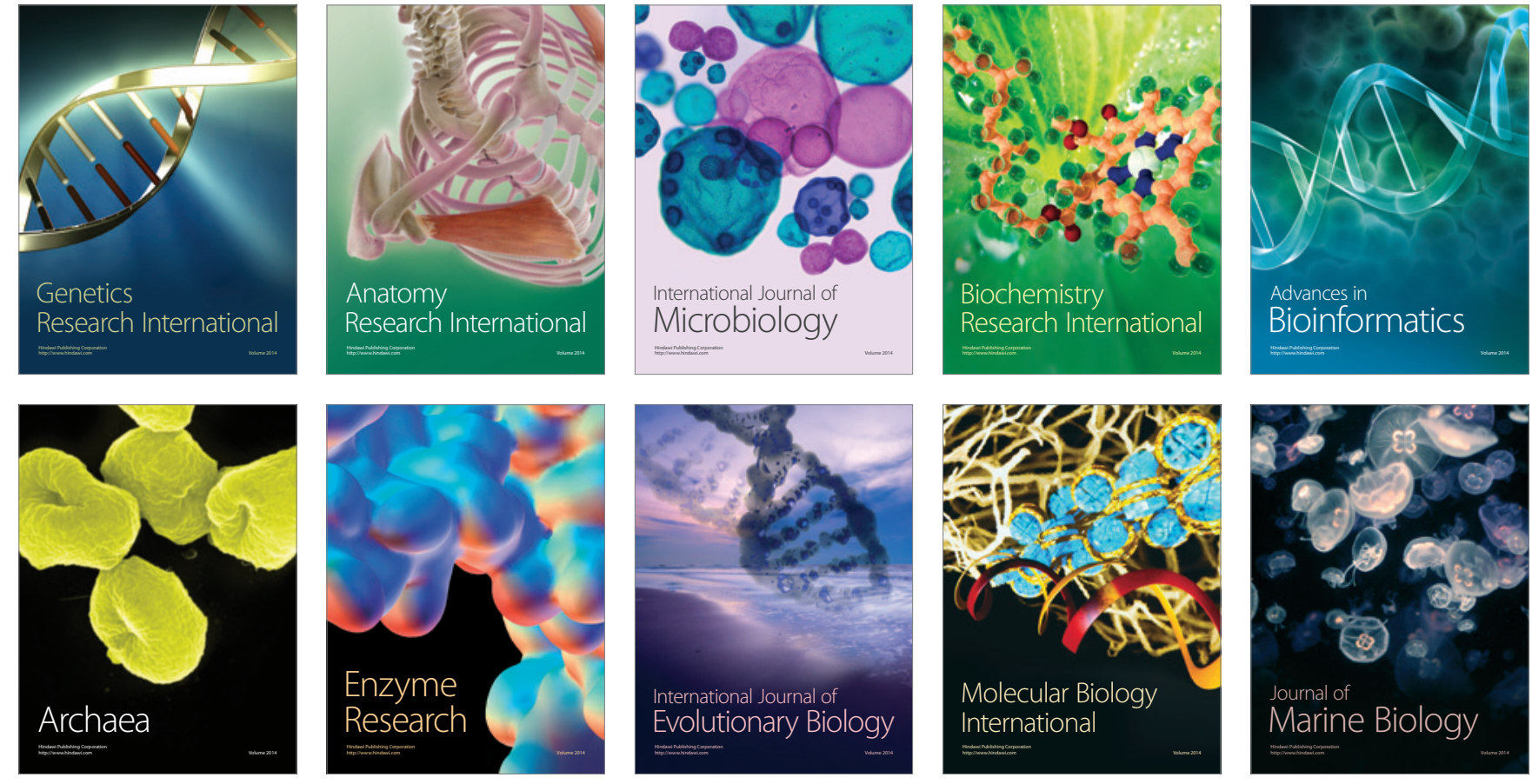